\title{
Design and Implementation of the Integration Information Platform of the Intravenous Therapy Safety and Management
}

\author{
Hong Wang ${ }^{1+}$, Jun $\mathrm{Bi}^{2}$, Zhiyun Zhao ${ }^{1}$ \\ ${ }^{1}$ Information Department, The 309th Hospital of PLA \\ ${ }^{2}$ Institute for Network Sciences and Cyberspace, Tsinghua University \\ ${ }^{2}$ Department of Computer Science, Tsinghua University \\ ${ }^{2}$ Beijing National Research Center for Information Science and Technology (BNRist)
}

\begin{abstract}
The paper designs and implements the integration information platform of the intravenous therapy safety and management. The platform is based on the $\mathrm{B} / \mathrm{S}$ structure with the development tool Eclipse and the database MySQL to implement the following five functions: to give publicity to the relative knowledge on the intravenous therapy safety and management, to conduct PICC catheterization and complication management, to learn on line, to instantly communicate on line, and to conduct quality management. The platform optimizes the process of the intravenous therapy safety and management, and reduces the workloads and error rates of nurses, moreover, training and expertise sharing are convenient and efficient. The platform has been used by all nursing units of our hospital. The platform can effectively raise the level of the intravenous therapy safety and management, furthermore, it can be promoted to other hospitals, and therefore the platform has high economic value.
\end{abstract}

Keywords: intravenous therapy; safety and management; information platform

\section{Introduction}

Peripherally Inserted Central Catheter (PICC) is a common nursing operation, which can effectively protect the upper limb veins, reduce the occurrence of phlebitis, and relieve the pain of patients, improving the life qualities of patients [1]. In 2014, our hospital set up a nursing specialist clinic - PICC clinic, which took over the PICC catheterization about a thousand times a year, received intravenous therapy consultations hundreds of times a year, and conducted complications several tens of times a year in our hospital. PICC catheterization and complication treatment are widely used in the fields of tumor chemotherapy, irritant drug infusion, intravenous nutrition therapy and long-term intravenous infusion.

The manual management mode has been ever used in our hospital's PICC catheterization and complication management: an application department fills in the application form of Word format, and sends it to PICC clinic through Office Automation System (OA) of our hospital, and then the application department call the PICC clinic to confirm the receiving. Subsequently, PICC clinic conducts PICC catheterization and complication management, and fills in the consultation form of Word format, and then send it back to the application department through OA. In summary, (1) from the beginning of the application to the end of disposal, the business process is complicated and has a low efficiency. (2) the filling items of the application form and consultation form are complicated and it is easy to manually fill them wrongly. (3) the information statistics and analysis of PICC catheterization and complication management are completed manually, so it is time-consuming and laborious, and the information correlation degree is not enough, furthermore, it is difficult to trace back, and it is hard to monitor the quality of PICC catheterization

\footnotetext{
Corresponding author. Tel.: +86 13520558701; fax: +86 01066775855 .

E-mail address: thc@pku.org.cn.
} 
and complication management. In addition, our hospital also lacks a unified information platform of the intravenous therapy safety and management (includes the following functions: propaganda of the intravenous therapy safety and management, online communication, online learning, and quality management), which is unfavourable for the further development of the intravenous therapy in our hospital.

Based on the intranet of the hospital, this paper designs and implements a comprehensive information platform of the intravenous therapy safety and management based on the computer network technologies. The platform propagates the organization framework, system specifications and latest news related to the intravenous therapy safety and management. Through the platform, we can on line carry out the application, appointment, disposal, statistics and analysis of PICC catheterization and complication treatment, and we can also carry out questions and answers about the intravenous therapy safety and management, searches, online learning, online communication and quality management to improve the intravenous therapy safety and management in our hospital.

The rest of the paper is organized as follows: In Section 2, we describe system design of the integration information platform of the intravenous therapy safety and management. Section 3 introduces platform implementation. And in Section 4, the conclusions are given.

\section{System Design}

\subsection{Design Goal}

Based on the latest JSP, Swing, Servlet, JavaBean, Struts, MySQL database and the intranet of the hospital, this paper designs and implements a unified information platform for propagation of the intravenous therapy, PICC management information system, online learning, online communication and quality

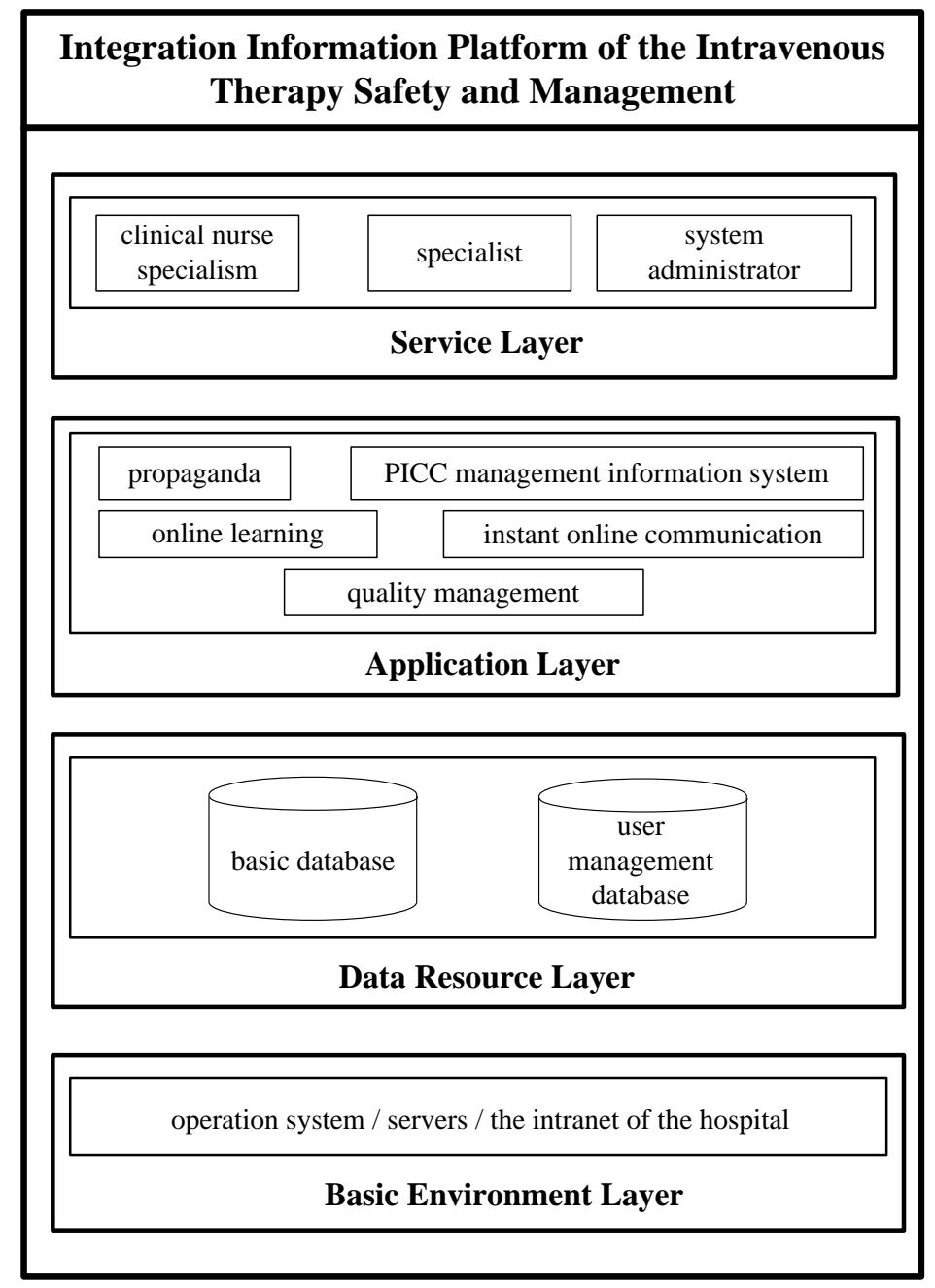

Fig. 1: the architecture of the integrated information platform for the intravenous therapy safety and management 
management, which can effectively improve the safety and management of intravenous therapy in our hospital.

The design objectives of the integration information platform of the intravenous therapy safety and management are as follows:

(1) to build a propaganda and learning platform for the intravenous therapy safety and management. Through the platform, we can publish the organization framework, system specifications, latest news and learning materials related to the intravenous therapy safety and management.

(2) to construct PICC management information system, which optimizes the application, appointment and disposal processes of PICC catheterization and complication management, realizes data inquiries under complex conditions, and carries out the strict authority and safety management on PICC catheterization and complication management.

(3) To build a communication platform for the intravenous therapy safety and management. Experts interact with nurses, and answer questions, promoting the continuous improvement of the theoretical knowledge and practical operation skills.

(4) The platform has good scalability. New functional modules can be embeded according to the continuous expansion of the demands for the intravenous therapy safety and management.

\subsection{Function Module Design}

The integrated information platform for the intravenous therapy safety and management is composed of five modules as follows: propaganda module, PICC management information system, online learning module, online communication module and quality management module. These five modules form an organic whole to realize the functions of the comprehensive information platform of the intravenous therapy safety and management.

(1) Propaganda module. The module is designed and implemented based on Swing and MySQL database. The system administrator can log in to the module, publish the profile of the vein study group, the curriculum vitaes of the members, the organization structure, the system specifications, the latest news (videoes of conferences and lectures) and so on. The relative data are stored in the MySQL database.

(2) PICC management information system. Combining with the characteristics of PICC catheterization and complication treatment, we optimize the processes of PICC catheterization and complication treatment, and use the most popular network technologies (JSP, Servlet and Struts) to develope a dynamic and interactive website, based on the intranet of the hospital. The website may be friendly and conveniently used. The application departments or PICC clinic use internet browsers to apply (appointment) or dispose PICC catheterization and complication treatment, and the related business data are stored in the backstage database, MySQL. The application departments and PICC clinic can conveniently and quickly make comprehensive information inquiries and statistics of PICC catheterization and complication treatment, which is the foundation of the next-step data mining.

(3) Online learning module. The module is based on JSP, JavaBean and MySQL database. The system administrator can $\log$ in to the online learning module, and publish the knowledge and exams of the the intravenous therapy, which users can download for learning and examination, respectively.

(4) Quality management module. The module is based on Servlet, JavaBean and MySQL database. The system administrator can $\log$ in to the quality management module, and publish the checklists of the intravenous therapy, the feedback tables of the vein study group and the self-examination feedback tables of the departments, which users can download, feed back and carry out online quality management.

(5) Online communication module. The module is based on Struts and MySQL database. The online communication module adopts the similar network technology to Bulletin Board System (BBS). Users do not need a formal username on the platform, and may anonymously ask questions and search the contents of the online communication module. The nursing department in our hospital arranges experts to take turns to answer users' questions. The experts and users interact with each other on the platform, improving the knowledge and understanding of the intravenous therapy safety and management. 


\section{Platform Implementation}

The platform, based on $\mathrm{B} / \mathrm{S}$ structure, is deployed in the intranet of our hospital. It is divided into the client and server side.

On the client side, clinical nurse specialisms for the intravenous therapy, the intravenous therapy experts, and system administrators use browsers in different identities to log in to the platform, which provides appropriate services for different identities.

The server side adopts three-tier hierarchical structure which is extensible: presentation layer, business logic processing layer, and data operation layer. The 3-tier hierarchical structure is shown in Figure 2. The hierarchical structure puts user interface, the business logic processing, data operation in the presentation layer, the business logic processing layer and the data operation layer, respectively. The client does not interact directly with the backstage database in order to ensure the security of the backstage data.

The presentation layer, which provides the User Interface (UI) for the clients, receives requests that the clients submit through browsers, and returns the results processed by the business logic processing layer to the clients. The business logic processing layer is the core of the three-tier structure, which is composed mainly of the JavaBean component [2]. JavaBean is a kind of Java class [3]. It is a kind of reusable components written in Java. By defining a series of attributes and providing access and modification methods to implement the encapsulation and reuse of data information [2]. The business logic processing layer receives the requests sent from the presentation layer to carry out business processing. In the meantime, the business logic processing layer accesses the data operation layer and the backstage database is operated. The data operation layer is mainly responsible to receive the data access requests from the business logic processing layer, and to manipulate the data, and then to return results to the business logic processing layer. The data operation layer manages the data including MySQL data, text files, binary files, XML files [4], Excel files [5] and so on. The clients can not directly access the data operation layer.

In the implementation of this platform, we use the Web server [6] to implement the functions of the presentation layer, the business logic processing layer and the data operation layer. We select the application server, Apache Tomcat 8 [7] as the Web server to respond to the various requests from the clients. The background database of the platform is the MySQL 5.5.57 [8], which is responsible for all the data involved in the platform. The data operation layer of Apache Tomcat 8 interacts with the MySQL 5.5.57 through the interface, Java DataBase Connectivity (JDBC) [9].

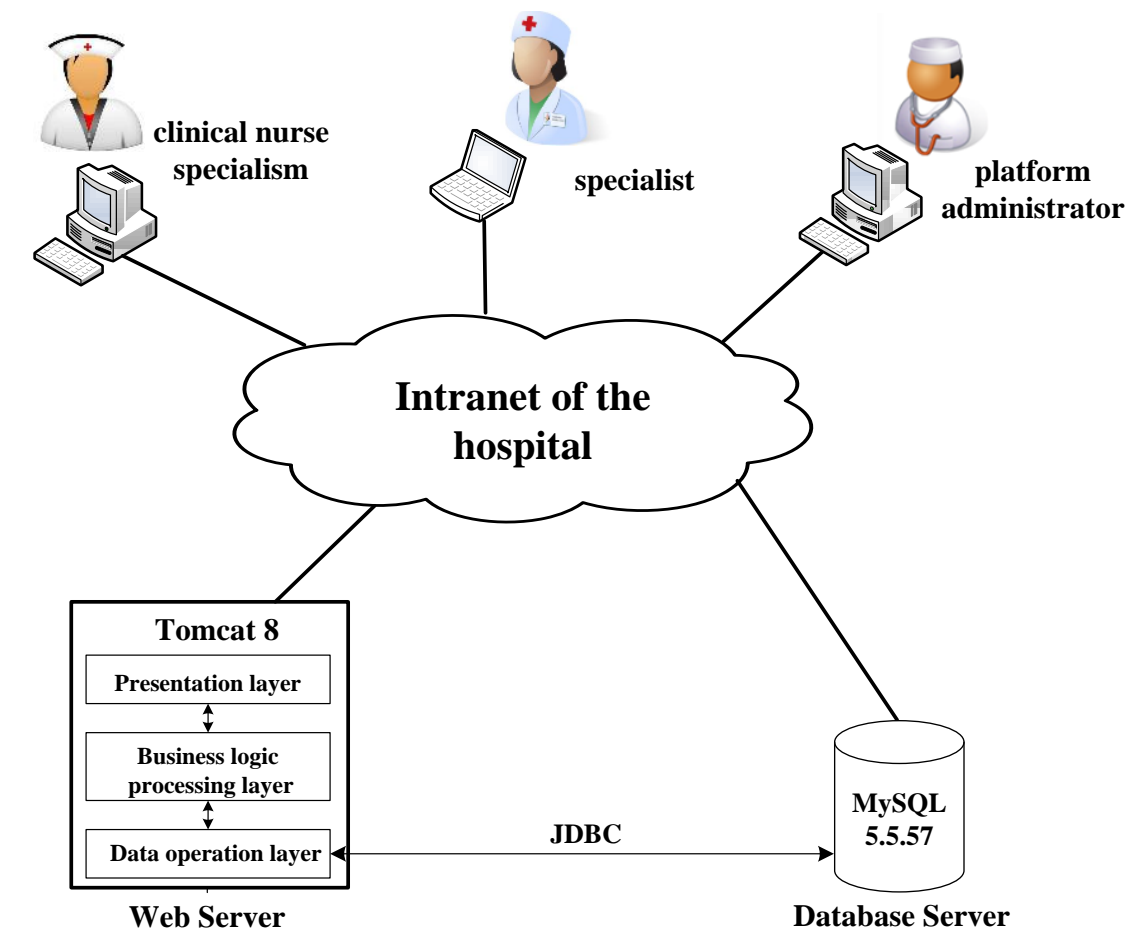

Fig. 2: 3-tier hierarchical structure of the integrated information platform of intravenous treatment safety and management 


\section{Conclusions}

This paper designs and implements a comprehensive information platform of the intravenous therapy safety and management. This platform propagates the organization framework, system specifications and latest news related to the intravenous therapy safety and management, and supports application, appointment, disposal, statistics and analysis of PICC catheterization and complication management, and then plunks for online learning, online communication and quality management of the intravenous therapy, effectively promoting the intravenous therapy safety and management. In the next step, we plan to integrate PVAS and intravenous infusion routes into the platform, further improving the platform functions.

\section{Acknowledgements}

This work is supported by National Key R\&D Program of China (2017YFB0801701), the National Science Foundation of China (No.61472213) and China Postdoctoral Science Foundation under Grant 2015M572723, the Key Hospital-level Project of the 309th Hospital of PLA (2017ZD-006) and the General Hospital-level Project of the 309th Hospital of PLA (2016MS-021).

\section{References}

[1] Baidu Wikipedia. Peripherally Inserted Central Catheter. https://baike.baidu.com/item/\%E7\%BB\%8F\%E5\%A4\%96\%E5\%91\%A8\%E9\%9D\%99\%E8\%84\%89\%E7\%A9\%B F\%E5\%88\%BA\%E4\%B8\%AD\%E5\%BF\%83\%E9\%9D\%99\%E8\%84\%89\%E7\%BD\%AE\%E7\%AE\%A1/1 103570 3? fr=aladdin, 2018.

[2] Bill Burke, Andrew Lee Rubinger. Enterprise JavaBeans 3.1: Developing Enterprise Java Components. O'Reilly Media, 2010.

[3] Richard Warburton. Java 8 Lambdas: Functional Programming for the Masses. Posts \& Telecom Press, 2015.

[4] Wikipedia. XML. https://en.wikipedia.org/wiki/XML, 2018.

[5] Excel Home. Excel 2013 function and formula application. Peking University Press, 2016.

[6] Wikipedia. Web server. https://en.wikipedia.org/wiki/Web_server, 2018.

[7] The Apache Software Foundation. Apache Tomcat8. http://tomcat.apache.org/tomcat-8.0-doc/index.html, 2018.

[8] Oracle Corporation. Download MySQL Community Server. https://dev.mysql.com/downloads/mysql/5.5.html, 2018.

[9] Yang Yuan. An introduction to the Jbuilder 6 instances. China Youth Publishing Group, 2002. 